\title{
Incidencia de la mecanización del suelo sobre sus propiedades físicas y el crecimiento vegetativo de papaya (Carica papaya L.)
}

\author{
Incidence of the soil mechanization over the physical properties and the vegetative \\ growth of papaya (Carica papaya L.)
}

\section{Diego Fernando Mendez V. ${ }^{1}$, Luís Carlos Becerra 0. ${ }^{2}$; Héctor Eduardo Hernández N. ${ }^{{ }^{*}}$, Anthony José Díaz I. ${ }^{4}$, Alfonso Julián Muñoz $\mathbf{M}^{5}$.}

\begin{abstract}
1. Ingeniero Agroecólogo, Universidad de la Amazonia, Florencia, Colombia, diegofdo9101@hotmail.com. 2. Ingeniero Agroecólogo, Universidad de la Amazonia, Florencia, Colombia, luiscarlosmf92@gmail.com.

3. Ingeniero Agroecólogo, Universidad de la Amazonia, Florencia, Colombia, eduardo.5560@hotmail.com 4. Ingeniero Agroecólogo, Universidad de la Amazonia, Florencia, Colombia, antonyjd9@gmail.com.

5. Ingeniero Agroecólogo, Universidad de la Amazonia, Florencia, Colombia.
\end{abstract}

Citar: MENDEZ, D.; BECERRA, L.; HERNANDEZ, H.; DIAZ, A.; MUÑOZ, A. 2016. Incidencia de la mecanización del suelo en el crecimiento vegetativo de papaya (Carica papaya L.). Rev. Cienc. Agr. 33(1):53-63.

Fecha de recepción: Enero 25 de $2016 . \quad$ Fecha de aceptación: Marzo 14 de 2016.

\begin{abstract}
RESUMEN
Para determinar la incidencia de la mecanización del suelo sobre sus propiedades físicas y el crecimiento vegetativo de papaya en Florencia (Caquetá), se utilizó el Diseño de Bloques Completos al Azar con tres tratamientos: suelo mecanizado con Motoazada, Azadón y no mecanizado - Testigo y cuatro repeticiones. Se midió en campo la resistencia a la penetración y los índices de crecimiento vegetativo, mientras que en laboratorio se calcularon la porosidad total, y la densidad aparente. En el mes uno $\left(\mathrm{M}_{1}\right)$ la resistencia a la penetración presentó diferencias significativas $(\mathrm{p}<0,05)$ para la profundidad de $0-10 \mathrm{~cm}$. La densidad aparente presento diferencias significativas $(\mathrm{p}<0,05)$ en las profundidades de $0-10$ y de $10-20 \mathrm{~cm}$ entre SMMTA con relación a SMAZ y testigo que no presentaron diferencias significativas $(\mathrm{p}>0,05)$ en ninguna de las profundidades. La densidad aparente y resistencia a la penetración aumentaron a través del tiempo. Los mayores valores de producción de asimilados y rendimiento en adsorción de energía lumínica representada en la tasa de asimilación neta, se presentaron a los 180 días después de la siembra en Testigo, donde alcanzó $10,04 \mathrm{gm}^{-2} \mathrm{dia}^{-1}$. La duración de área foliar y la tasa de crecimiento del cultivo fueron las variables más sensibles en respuesta de los cambios de porosidad y resistencia a la penetración del suelo. El Testigo, alcanzó los mayores niveles de tasa de asimilación neta, posiblemente por la homogeneidad en la ganancia de asimilados, a través del periodo fotosintético evaluado.
\end{abstract}

Palabras claves: asimilación, desarrollo, crecimiento, rendimiento. 


\begin{abstract}
In order to determine the incidence of the soil mechanization over its physical properties and the vegetative growth of papaya in Florencia (Caquetá), it was used a randomized block design with three treatments: mechanized soil with motor-hoe, hoe and no mechanized - control with four repetitions each. The resistance to the penetration, and vegetative growth indexes were measured in the field while the total porosity and apparent density were calculated in the lab. In the first month (M1) the resistance to the penetration showed significant difference $(p<0,05)$ to $0-10 \mathrm{~cm}$ depth. The apparent density presented significant difference $(p<0,05)$ to $0-10$ and $10-20 \mathrm{~cm}$ depth between SMMTA related to SMAS and no mechanized, which did not exhibit significant differences at any depth. The apparent density and the resistance to the penetration increased with the time. The greatest values of production assimilates and light energy absorption performance represented in the net assimilation rate, were observed in the witness 180-days after planting, when it reached 10,04 gm-2day-1. The duration of the leaf area and growth rate of the crop were the variable more sensitive to changes of the porosity and resistance to the penetration of the soil. The witness treatment reached the highest levels of net assimilation rate, probably due to the homogeneity of the assimilated gains through the photosynthetic time evaluated.
\end{abstract}

Key words: assimilation, development, growth, performance.

\section{INTRODUCCIÓN}

Con el uso agropecuario tradicional, se transforma la arquitectura de la vegetación natural y se afecta de manera substancial la composición, la estructura y el funcionamiento del ecosistema; esto conlleva no sólo a que desaparezcan los mecanismos de conservación y de fertilidad natural de los suelos, sino también a que se aceleren los procesos de degradación de la áreas intervenidas o colonizadas (Escobar et al., 1998). En el caso del departamento del Caquetá, su territorio presenta un 27,77\% de área intervenida, de las cuales la actividad ganadera, representa 27,08\% (Beltrán y Torrijo, 2013).

Pinzón y Amézquita (1991) aseguran, que el establecimiento de pasturas en el bosque húmedo tropical junto con el pisoteo de los animales, favorecen la compactación del suelo, condición que reduce el volumen de los poros de mayor diámetro, lo que ocasiona cambios en el contenido de humedad y en el intercambio de gases entre el suelo y la atmosfera, e impide, además, el desarrollo de las raíces. La percepción sobre la existencia de compactación y endurecimiento del suelo resultado de actividades productivas, ha llevado a proponer la disrupción mecánica de capas compactadas con implementos de labranza y mecanización (Álva- rez et al., 2006; Álvarez et al., 2009), estas actividades mejoran los parámetros físicos del suelo en mención y condicionan una mayor respuesta de producción en plantaciones (Soza et al., 2006). Por lo que es necesario preparar los suelos para la siembra mediante las labores de mecanización (Arroyo et al., 2003). Logrando cambiar su estructura (García, 2006), disminuyendo la resistencia a la penetración y la densidad aparente (Álvarez et al., 2009), de tal manera que facilite tanto la germinación optima de semillas como la penetración de las raíces, y finalmente el desarrollo productivo de los cultivos (García, 2006).

La papaya (Carica papaya L.) es uno de los cultivos frutícolas de importancia económica y social en las regiones tropicales y subtropicales del mundo (Escamilla et al., 2003), en los últimos cinco años ha tenido un crecimiento productivo a nivel mundial (Díaz et al., 2014). En Colombia se encuentra entre las principales frutas cultivadas junto con el banano y los cítricos (Hernández, 2009). La planta es de rápido crecimiento con una producción temprana y continua, el cultivo es afectado por diversos factores de origen biótico y abiótico, los cuales tienen un efecto significativo sobre el vigor y la sanidad de la planta, así como en el rendimiento y la calidad del fruto (Escamilla 
et al., 2003), muestra gran sensibilidad, a sufrir daños en sus raíces y descensos de rendimientos a causa de la compactación (Yamanishi et al., 1998; Campostrini y Yamanishi, 2001), estos mismos autores en estudios realizados encontraron que las plantas de papaya sometidas a compactación del suelo; redujeron el número total de hojas, el área foliar promedio, la longitud de la hoja de la vena central, área foliar total, diámetro del tronco y altura de las plantas, con relación a las plantas no restringidas.

En este contexto, se analizó la incidencia de la mecanización del suelo sobre sus propiedades físicas y el crecimiento vegetativo de papaya (Carica papaya L.), en el centro de investigaciones Amazónicas Macagual - Cesar Augusto Estrada González, Florencia, Caquetá.

\section{MATERIALES Y MÉTODOS}

El trabajo, se desarrolló en el Centro de Investigaciones Amazónicas Macagual - Cesar Augusto Estrada González de la Universidad de la Amazonia, el cual se encuentra ubicado en el municipio de Florencia, del Caquetá, Colombia, a $1^{\circ} 37^{\prime} \mathrm{LN}$ y $75^{\circ} 36^{\prime} \mathrm{LO}$ a 300 msnm, con una temperatura media de $25,5^{\circ} \mathrm{C}$, humedad relativa media del $85 \%$, precipitación media anuales de $3600 \mathrm{~mm} \cdot \mathrm{año}^{-1}$, brillo solar de 1707 horas $\cdot a n ̃ 0^{-1}$, suelos ultisoles (Trujillo et al., 2012).

En un área de $2.204 \mathrm{~m}^{2}$, se utilizó un Diseño de Bloques Completos al Azar con tres tratamientos y cuatro repeticiones, para un total de 12 parcelas. Se utilizaron plantas de papaya hibrido Tainung-01. Bajo las recomendaciones de Jiménez (2002), se sembró la papaya a una distancia de 2 metros entre planta y 1,8 metros entre surco, para un total de 36 plantas por unidad experimental y 432 plantas en el diseño. Los tratamientos a avaluar fueron: mecanización del suelo con moto azada (SMMTA), mecanización con azadón (SMAZ) y Testigo; este último sin presentar procesos de mecanizado en el suelo.
Posterior a la preparación del suelo (mes uno) y quince meses después (mes quince), en las profundidades de $0-10 \mathrm{~cm}$ y $10-20 \mathrm{~cm}$ (Bravo y Andreu, 1995), se analizaron los parámetros: Densidad real, método del picnómetro (Torrente y Valenzuela, 2013), densidad aparente (DA), método del cilindro (Montenegro y Malangón, 1990), resistencia a la penetración (RP), usando el Penetrómetro manual Eijkelkamp, humedad gravimétrica (HG), mediante secado en estufa a $105^{\circ} \mathrm{C}$ (Montenegro y Malangón, 1990) y porosidad total (PT), mediante las fórmula utilizada por Torrente y Valenzuela (2013).

Para registrar la dinámica de producción de materia seca (MS), se realizaron muestreos destructivos cada 45 días, hasta los 225 días después de la siembra (dds). Se midió área del suelo (AS) con relación al área que ocupa la planta. Se colectaron dos plantas por unidad experimental para un total de ocho plantas por tratamiento. A cada planta se le separaron por estructuras vegetativas y fruto. Posterior a ello, se recubrió con bolsa de papel por separado y se sometieron a secado en horno eléctrico a temperatura de $105^{\circ} \mathrm{C}$, durante 48 horas para el muestreo uno y dos, 60 horas para el muestreo tres y cuatro, por último 84 horas para el muestreo cinco, la suma de estos pesos, representó el peso de MS total por planta. El área foliar se determinó con el programa ImageJ® (Gil et al., 2014). A partir de los valores de MS total, área foliar, área de suelo y tiempo entre muestreos, mediante las formulas propuestas por Barrera et al. (2010), se calculó el índice de área foliar (IAF), duración de área foliar (DAF), tasa de asimilación neta (TAN), tasa de crecimiento del cultivo (TCC), tasa de crecimiento relativo (TCR).

Se realizó el Análisis de Varianza (ANDEVA) en las variables seleccionadas, a nivel de propiedades físicas del suelo e índices de crecimiento vegetativo. Las medias se compararon con el test LSD Fisher, con una confiabilidad del 95\%, mediante el programa estadístico InfoStat (Di Rienzo et al., 2014). 


\section{RESULTADOS Y DISCUSION}

Los valores de porosidad presentaron diferencias significativas en el mes uno, entre SMAZ y Testigo respecto al SMMTA (Tabla 1). El SMMTA y SMAZ incrementaron los valores de porosidad en un 10,48 y 3,09\%, respectivamente, en la profundidad de $0-10 \mathrm{~cm}$ respecto al Testigo, en la profundidad de $10-20 \mathrm{~cm}$ el aumento del SMAZ y el SMMTA fue de 9,35 y 1,35\%. Los aumentos en la porosidad generan mayor aireación y humedad del suelo (Botta et al., 2007). Las impedancias mecánicas del suelo, medidas a través de la RP, disminuyeron por efecto de la mecanización, presentándose diferencias significativas entre los tres tratamientos en el tiempo uno como se muestra en el (Tabla 1). En la profundidad de 0-10 cm en SMMTA y SMAZ presentaron una disminución en la RP de 85,50 y 62,31\% en su orden respecto al testigo. En la profundidad de $10-20 \mathrm{~cm}$ la disminución fue de $57,65 \%$ para SMMTA y 28,82\% SMAZ respecto al testigo, resultados similares fueron reportados por Álvarez et al. (2006) que en los primeros $25 \mathrm{~cm}$ del suelo registraron disminuciones de RP en $37 \%$ después de mecanizado el suelo. Los tres tratamientos no presentaron problemas de compactación, según los siguientes umbrales propuestos por Álvarez et al. (2009) de 1,5 MPa (50\% de disminución en el crecimiento radical de maíz) a $3 \mathrm{MPa}$ (detenimiento del crecimiento radical), aunque estos valores varían según las condiciones fisiológicas del cultivo,
Jorajuria y Draghi (2000) encontraron niveles críticos de RP>2,0Mpa y Díaz (2004) reporta niveles críticos de RP entre 1,22 y 2,00MPa (prof. $<0,20 \mathrm{~m}$ ). Se presentó un incremento significativo $(\mathrm{P}<0,05)$ de RP del tiempo uno al tiempo dos en SMMTA en las profundidades de 0-10 y $10-20 \mathrm{~cm}$ y en SMAZ en las profundidades de 0-10 concordando con lo encontrado por Elissondo et al. (2001). El mayor aumento de RP en las diferentes profundidades lo presento SMMTA. En la profundidad de $10-20 \mathrm{~cm}$ igualó los valores de RP del tiempo uno del testigo (Tabla 1). Este comportamiento puede ocurrir por diferentes razones, entre ellas, el proceso reordenamiento de las partículas del suelo, tal como lo señalan Bravo y Andreu (1995), por las gotas de lluvia debido que en el momento que caen en la tierra desintegra los agregados, dispersa la arcilla (Richart et al., 2005), generando una película de agua que obliga a infiltrar las fracciones texturales, primero arena, posteriormente limos y arcilla (Becerra et al., 2005). Aunque la RP en el testigo no presento diferencias significativas entre el tiempo uno y tiempo dos en la profundidad de 0-10cm, hubo una mínima disminución de $0,69 \mathrm{MPa}$ a $0,58 \mathrm{MPa}$, coincidiendo con resultados encontrados por Elissondo et al. (2001), pero diferente a lo reportado por Taboada (1998) que encontraró que cuando no se mecanizo el suelo, aumento la resistencia a la penetración en los primeros cm después de un tiempo.

Tabla 1. Dinámica de la porosidad (PT) y la resistencia a la penetración (RP), bajo diferente mecanización del suelo entre el mes uno y mes quince.

\begin{tabular}{lcrrrr}
\hline \multirow{2}{*}{ Trat. } & \multirow{2}{*}{ Prof. } & \multicolumn{2}{c}{ PT (\%) } & \multicolumn{2}{c}{ RP (MPa) } \\
\cline { 3 - 6 } & & Mes uno & Mes quince & Mes uno & Mes quince \\
\hline SMMTA & \multirow{2}{*}{$0-10$} & $65,03 \pm 1,05 \mathrm{~A} \mathrm{a}$ & $57,11 \pm 1,10 \mathrm{~B} \mathrm{~b}$ & $0,69 \pm 0,04 \mathrm{~A} \mathrm{a}$ & $0,58 \pm 0,05 \mathrm{~A} \mathrm{a}$ \\
SMAZ & & $58,81 \pm 1,02 \mathrm{~B} \mathrm{a}$ & $56,91 \pm 1,10 \mathrm{~B} \mathrm{~b}$ & $0,26 \pm 0,04 \mathrm{~B} \mathrm{~b}$ & $0,55 \pm 0,05 \mathrm{~A} \mathrm{a}$ \\
Testigo & \multirow{2}{*}{$10-20$} & $55,81 \pm 1,44 \mathrm{~A} \mathrm{a}$ & $47,52 \pm 1,51 \mathrm{~A} \mathrm{~b}$ & $1,11 \pm 0,09 \mathrm{~A} \mathrm{a}$ & $1,09 \pm 0,07 \mathrm{~A} \mathrm{a}$ \\
\hline SMMTA & $51,17 \pm \mathrm{B} \mathrm{a}$ & $45,52 \pm 1,51 \mathrm{~A} \mathrm{~b}$ & $0,79 \pm 0,09 \mathrm{~B} \mathrm{a}$ & $1,02 \pm 0,07 \mathrm{~A} \mathrm{a}$ \\
SMAZ & & $50,59 \pm 1,44 \mathrm{~B} \mathrm{a}$ & $48,50 \pm 1,51 \mathrm{~A} \mathrm{a}$ & $0,47 \pm 0,09 \mathrm{C} \mathrm{b}$ & $1,10 \pm 0,07 \mathrm{~A} \mathrm{a}$ \\
Testigo & & &
\end{tabular}

Trat: Tratamiento, Prof: Profundidad, letras en minúsculas distintas indican diferencias significativas entre filas $(\mathrm{p}<=0,05)$, letras en mayúscula distintas indican diferencias significativas entre columnas $(\mathrm{p}<=0,05)$ 
Los cambios registrados en la Da del suelo antes y después de la implementación del cultivo de papaya, se presentan en la Tabla 2. Después de aplicados los tratamientos la Da en el tiempo uno presento diferencias significativas entre SMMTA con respecto a SMAZ y testigo en las profundidades de 0-10 y 10-20 (Tabla 2), presentado disminuciones de 15,95\% el SMMTA con respecto al testigo en la profundidad de $0-10 \mathrm{~cm}$ y de $11 \%$ en las profundidades de 10-20 en los mismos tratamientos. Entre SMAZ y testigo aunque no se presentaron diferencias significativas disminuyó la Da en las profundidades de $0-10$ y $10-20 \mathrm{~cm}$ (Tabla 2), coincidiendo con resultados obtenidos por Álvarez et al. (2006). Los valores de Da registrados entre el tiempo uno y el tiempo dos para SMMTA y SMAZ presentaron diferencias significativas (Tabla 2), aumentando la Da a través del tiempo coincidiendo con resultados obtenidos por Bravo y Andreu (1995). El aumento del tiempo uno con respecto al tiempo dos en la profundidad de 0-10cm para el SMMTA fue de 19,38\% y para SMAZ fue de 9,18\%. En la profundidad de $10-20 \mathrm{~cm}$ el aumento en SMMTA fue $16,37 \%$ y para SMAZ fue de 10,83\%. La Da en testigo presento un leve cambio, pero sin diferencias significativas en ninguna de las profundidades, resultados similares encontrados por Álvarez et al. (2009). Los cambios fueron aumentos leves de Da en las profundidades de $0-10$ y $10-20 \mathrm{~cm}$. Comparando los datos de Da y RP, esta última presentó diferencias significativas entre los tres tratamientos (Tabla 1) a diferencia de la Da (Tabla 2), corroborando lo encontrado por Álvarez et al. (2009) donde demuestra una baja sensibilidad de este parámetro a los distintos manejos y afirmando lo planteado por Martínez et al. (2010), quienes afirman que la resistencia a la penetración es un parámetro más sensible que la densidad aparente para caracterizar la compactación. SMMTA si presentó diferencias significativas $(\mathrm{P}<0,05)$ con respecto a SMTA y testigo en las profundidades de $0-10$ y $10-20 \mathrm{~cm}$.

El comportamiento del IAF, bajo diferentes procesos de mecanización del suelo, para el periodo que comprende de los 45-180dds, fue de tendencia decreciente en los tres tratamientos y ascendente para el periodo final del muestreo de los 180-225dds, tal como se indica en la Figura 1. Las plantas del SMMTA mostraron el mayor IAF del ciclo evaluado y en relación con las plantas del SMAZ y Testigo; esta condición se registró solo para el inicio del periodo evaluado, aunque en ninguno de los tiempos se evidenciaron diferencias significativas $(\mathrm{P}<0,05)$ entre muestreos según el ANDEVA.

La DAF expresado en $\mathrm{cm}$ dia $^{-1}$, presentó un comportamiento inicial de decrecimiento entre los 90-180dds y ascendente para el final del ciclo evaluado entre los 180-225dds, como se indica en la Figura 2. Ningún tratamiento presento diferencias significativas.

Tabla 2. Dinámica de la densidad aparente bajo diferente mecanización del suelo entre el mes uno y quince.

\begin{tabular}{lccccccccc}
\hline & & \multicolumn{6}{c}{ Densidad aparente $\left(\mathrm{gr} / \mathrm{cm}^{3}\right)$} \\
\cline { 3 - 8 } Tratamiento & $\begin{array}{c}\text { Profundidad } \\
\text { (cm) }\end{array}$ & \multicolumn{9}{c}{ Tiempo } \\
\cline { 3 - 9 } & & & \multicolumn{6}{c}{ Mes uno } \\
TESTIG0 & \multirow{2}{*}{$0-10$} & 0,94 & \pm & 0,02 & $\mathrm{~A} \mathrm{a}$ & 0,89 & \pm & 0,03 & $\mathrm{~B} \mathrm{a}$ \\
SMAZ & & 0,89 & \pm & 0,02 & $\mathrm{~A} \mathrm{~b}$ & 0,98 & \pm & 0,03 & $\mathrm{~A} \mathrm{a}$ \\
SMMTA & & 0,79 & \pm & 0,02 & $\mathrm{~B} \mathrm{a}$ & 0,98 & \pm & 0,03 & $\mathrm{~A} \mathrm{a}$ \\
TESTIG0 & \multirow{2}{*}{ oct-20 } & 1,09 & \pm & 0,03 & $\mathrm{~A} \mathrm{a}$ & 1,13 & \pm & 0,03 & $\mathrm{~A} \mathrm{a}$ \\
SMAZ & 1,07 & \pm & 0,03 & $\mathrm{~A} \mathrm{~b}$ & 1,2 & \pm & 0,03 & $\mathrm{~A} \mathrm{a}$ \\
\hline
\end{tabular}




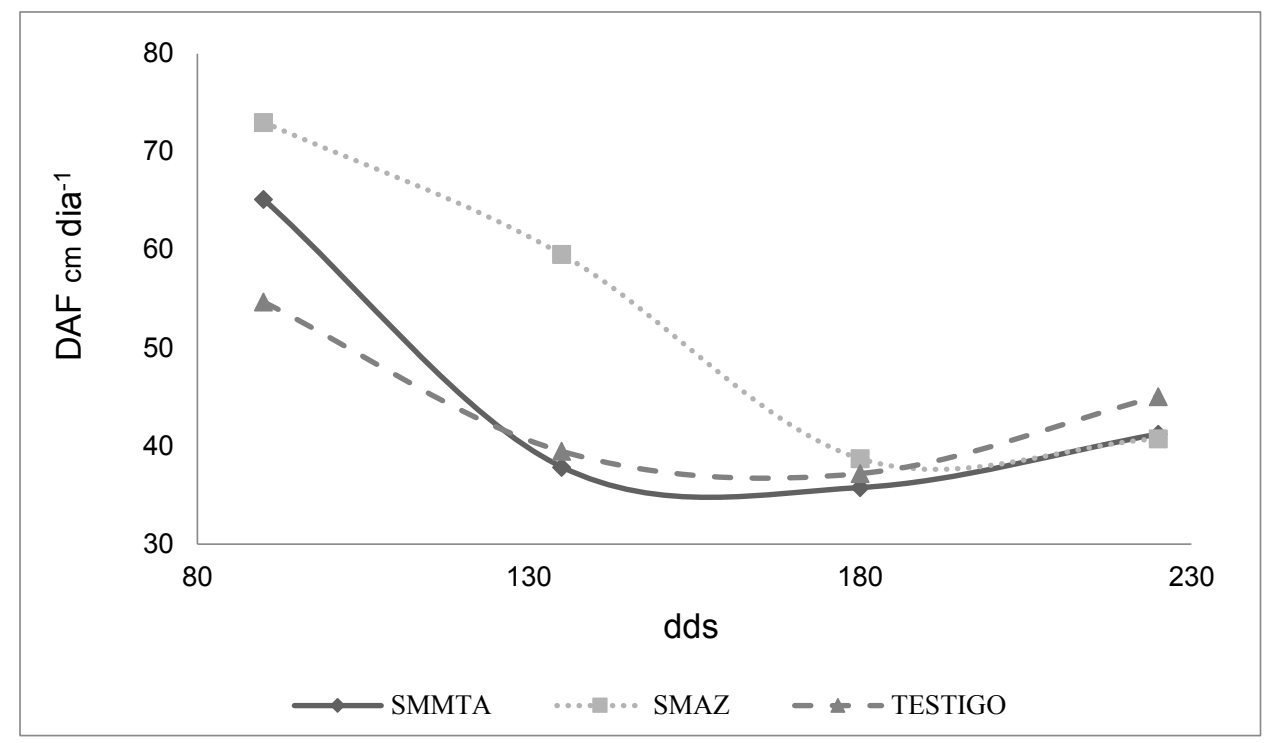

Figura 1. Índice de área foliar (IAF) de plantas de papaya sembrada bajo diferentes tipos de mecanización del suelo.

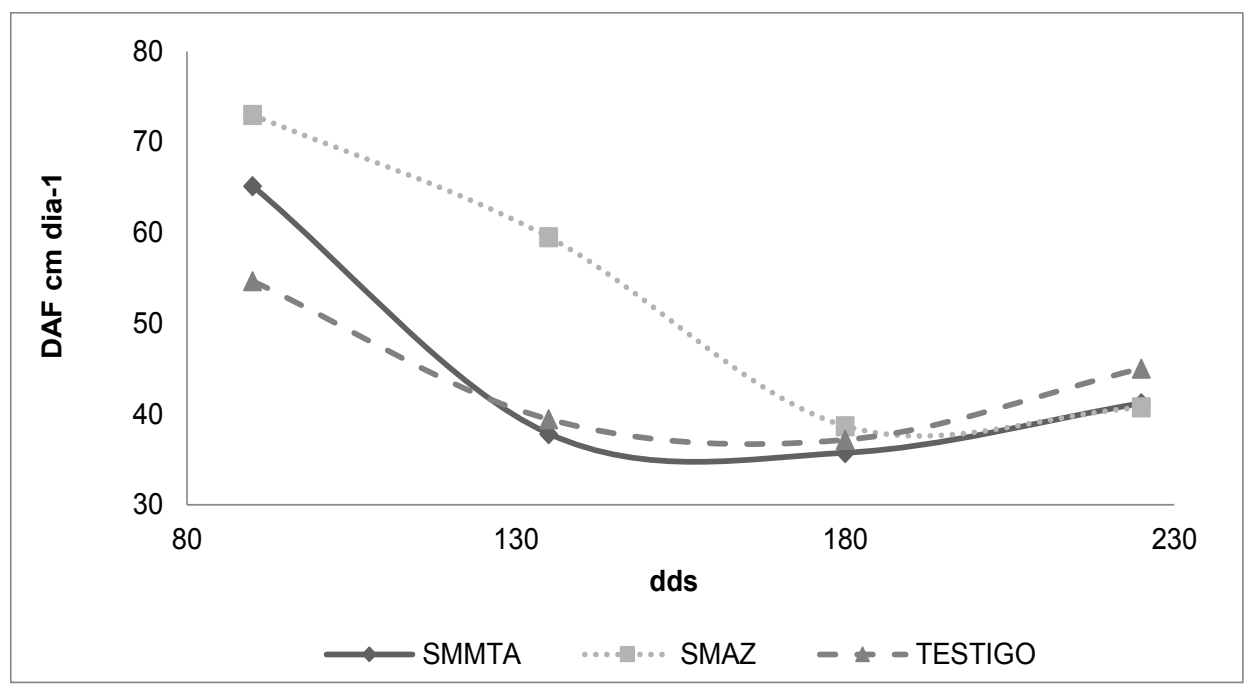

Figura 2. Duración área foliar (DAF) de plantas de papaya sembrada bajo diferentes tipos de mecanización del suelo.

En la Figura 3, se presenta la TCC en g m-2 dia-1. No se registran diferencias significativas entre los tratamientos para el incremento de materia en la planta por unidad de área del suelo y unidad de tiempo (Borrero et al., 2000), pero si un comportamiento irregular durante el ciclo que comprende de los 90225 dds, tiempo en el cual se generaron intervalos de ganancia y perdida de materia. Rasgos equivalentes son reportados por Barraza et al. (2004), Gil y Medina, (2007), en estudios adelantados con Lycopersicon esculentumen y Carica papaya, en donde la TCC disminuye y es constante por un periodo hasta alcanzar un mínimo, y posteriormente este índice crece en forma exponencial en intervalos de días; situación que asocia Rodríguez et al. (2003), con el potencial genético de las plantas y las condiciones ambientales que afectan la velocidad del crecimiento vegetal. 


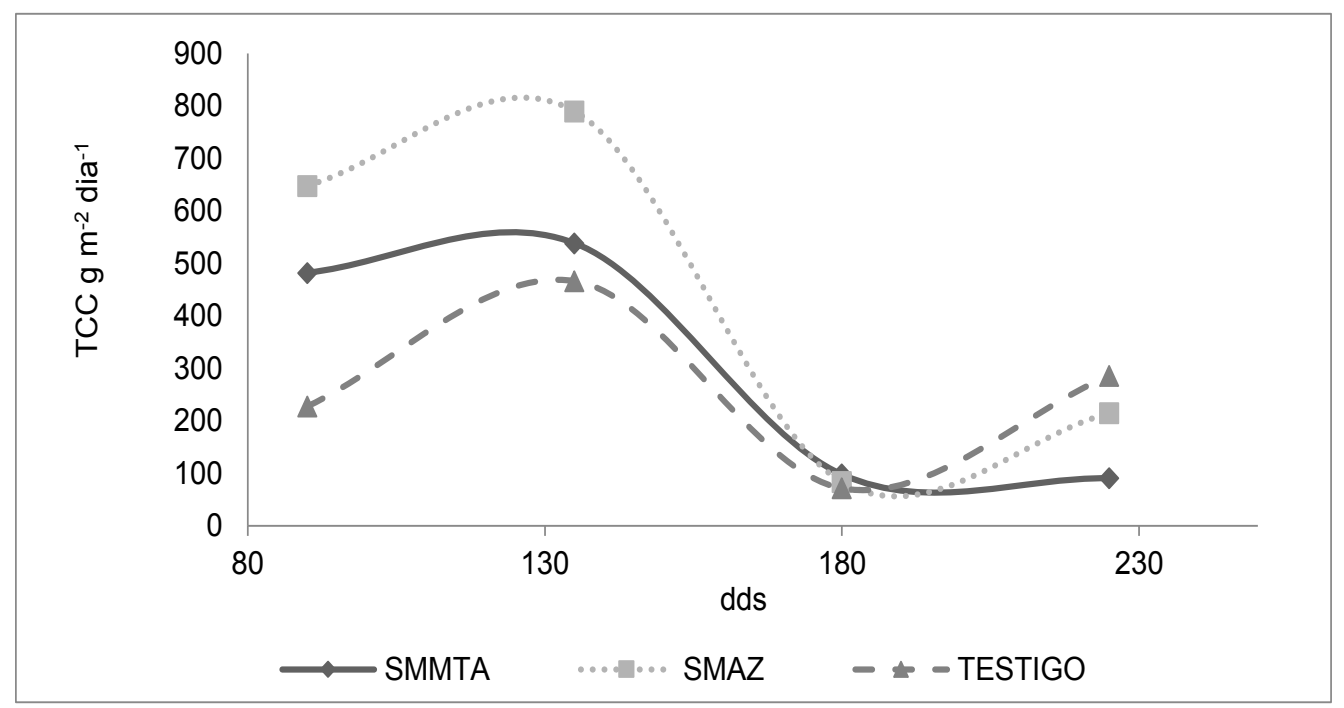

Figura 3. Tasa de crecimiento del cultivo (TCC) en plantas de papaya sembrada bajo diferente tipo de mecanización del suelo.

En la Figura 4, se presenta la TCR en $\mathrm{g} \mathrm{kg} \mathrm{dia}^{-1}$.El comportamiento inicial fue ascendente en el periodo que comprende de los 90-135dds y descendiente entre los 135-225dds. Bajo ninguno de los tratamientos se presentaron diferencias significativas $(\mathrm{p}<0,05)$ de acuerdo con los ciclos evaluados.

Para los tres tratamientos, se generó una acumulación positiva de TCR, entre los 90-135dds, condición que depende de la ganancia de materia seca en el tiempo (Gil y Miranda, 2007). Los mayores valores de TCR en éste ciclo fueron registrados por Testigo y SMAZ donde se alcanzó 20,91 g kg dia ${ }^{-1}$ para los 90 dds y 39,01 $\mathrm{g} \mathrm{kg}^{-1} \mathrm{dia}^{-1}$ en 135dds. Durante los 180-225dds, correspondiente a las dos últimas etapas de muestreo, los tratamientos con mejores respuestas, fueron Testigo $(27,99)$ y SMAZ $(12,37)$. Coincide con los ciclos iniciales, aunque, en esta ocasión los valores de almacenamiento decrecen de forma gradual.

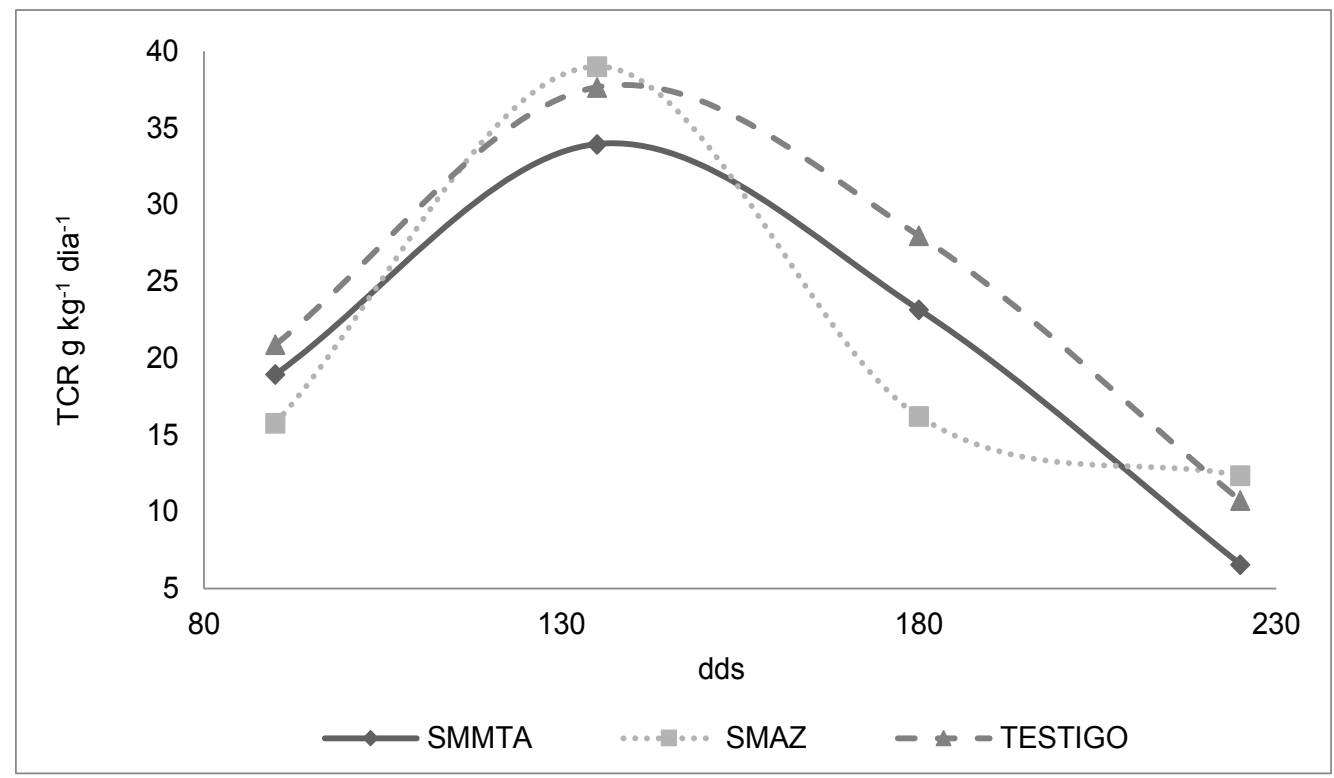

Figura 4. Tasa de crecimiento relativo (TCR) de plantas de papaya sembrada bajo diferente tipo de mecanización del suelo. 
La TAN, es un índice dependiente del peso seco y el área foliar expresado en $\mathrm{g} \mathrm{m}^{-2}$ dia $^{-1}$ (Gil y Miranda, 2007). No se presentaron diferencias significativas para la TAN del cultivo de papaya. El comportamiento en las plantas como sistema asimilatorio se observa en la Figura 5. Entre los 90-180dds, la TAN fue ascendente para los tres tratamientos, donde el SMMTA alcanza valores de 2,57 para los 90dds y Testigo 6,46 y 10,04 entre los 135-180dds correspondientes. Para el último periodo evaluado, comprendido entre los 180-225dds se evidenció un decrecimiento en la TAN, tiempo en el cual Testigo alcanza los mayores valores.

El IAF (Figura 1) fue mayor con relación al periodo comprendido entre los 45 y 56dds a diferencia de lo establecido por Gil y Miranda (2007), quienes evaluaron el crecimiento de papaya durante la fase de vivero, para las variedades de Maradol e híbrido Tainung- 1 , con valores de 0,42 y $0,31 \mathrm{~g} \mathrm{~m}^{-2}$ dia $^{-1}$, respectivamente. Aunque se aclara que los valores altos de este índice en algunas ocasiones, no están relacionados de manera directa con una mayor cantidad de fotosíntesis. El IAF es un índice que representa para todo cultivo, un promedio de los estratos de follaje que están expandidos, situación que se ve afectada por el hecho que las hojas no se desarro- llan sin dejar de encontrarse unas con otras, sino que lo hacen en diferentes ángulos que varían con la morfología de las especies y con las condiciones ambientales. Para corroborar esto, Barraza et al. (2004) indican que algunos valores altos del IAF, pueden ocurrir cuando el follaje del cultivo no cubre toda la superficie del suelo.

La dinámica descendente de la DAF expresada por los tratamientos para los 90-180 dds (Figura 2), se explica por lo planteado por Cayón (1992), al relacionar que la baja DAF en las plantas, obedece a una de las condiciones más comunes que contribuye al rápido envejecimiento de las hojas, ya sea por la escases o baja disponibilidad de nutrimentos, lo cual se torna limitante y genera que las hojas jóvenes se conviertan en un depósito preferencial.

Para los 180-225dds, se registra un incremento en la DAF, lo que representa según Santos et al. (2010) un mayor tamaño en el área foliar y en la persistencia a través del tiempo, además de una mejor acumulación de materia seca. Aguilar et al. (2005) corroboran que a mayor DAF, se puede producir un incremento en la producción de biomasa, debido a la mayor radiación interceptada por el dosel vegetal.

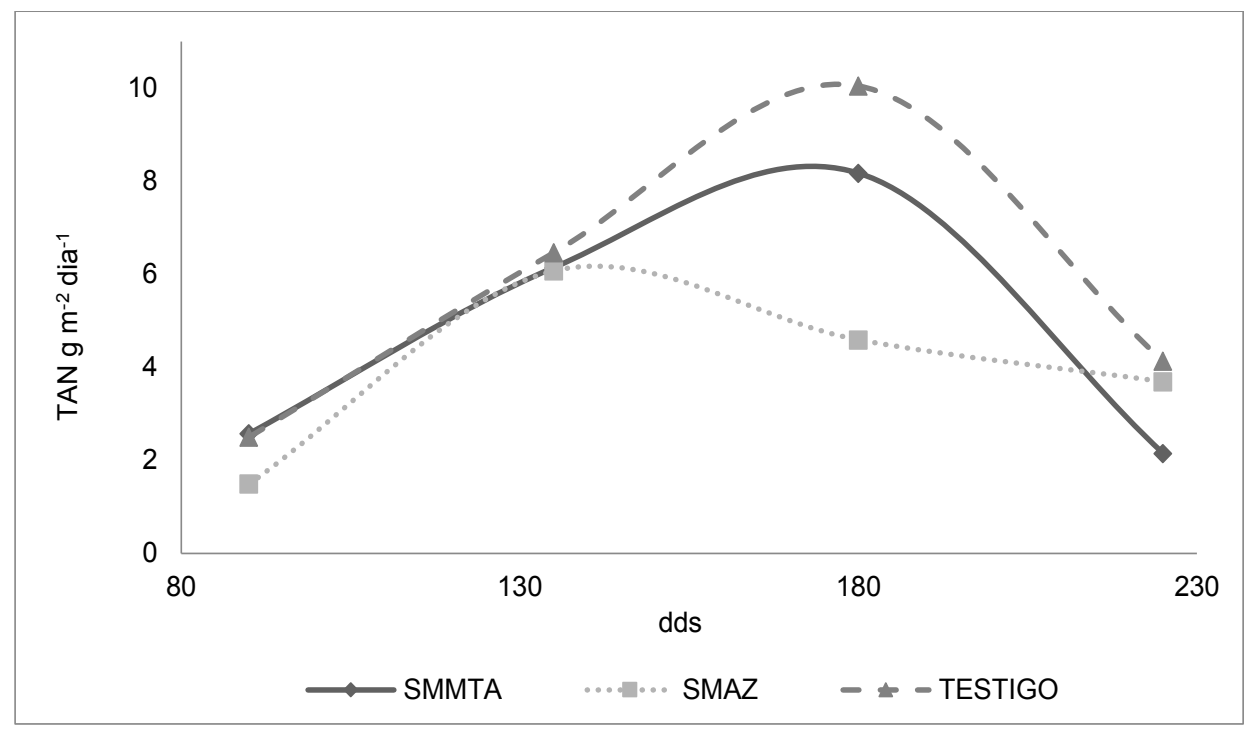

Figura 5. Tasa de asimilación neta (TAN) de plantas de papaya, sembrada bajo diferente tipo de mecanización del suelo. 
Los resultados obtenidos de la TCC (Figura 3), coinciden con los registrados por Barraza et al. (2004), Gil y Miranda (2007), en estudios adelantados en Lycopersicon esculentum P. Mill. y Carica papaya L., donde la TCC aumentó rápidamente hasta alcanzar un máximo, para posteriormente registrar la disminucion acelerada del índice de crecimiento, situación que interpreta y asocia Rodríguez et al. (2003), con el potencial genético de las plantas y las condiciones ambientales que afectan la velocidad del crecimiento vegetal.

En relación a la TCR (Figura 4), se puede comparar el primer comportamiento de los 90-135dds con las investigaciones adelantadas por Firmino et al. (2007), Gil y Miranda (2007), en plantaciones de papaya durante los 20 y 95dds, donde se presentaron dinámicas contrarias. La TCR se redujo de manera circunstancial a lo largo del periodo evaluado, inteRPretándose en un menor ingreso de materia seca en el tiempo.

El comportamiento general de la TCR entre los 45225dds puede explicarse de acuerdo con lo plateado por Jarma et al. (1999) al considerar que al inicio de la TCR en habichuela, se presentaron valores altos que fueron disminuyéndose conforme avanzó el ciclo de vida de las plantas. Nótese que en la plantación de papaya bajo los tres tratamientos, hubo una disminución de la TCR en el periodo comprendido entre los 135-225dds, tiempo a partir del cual, las plantas de Tainung-1 ya habían alcanzado su etapa de madurez e iniciado el proceso de floración. Es posible que esta situación, según Barraza et al. (2004), también obedezca a la fenología de la planta, debido a que las hojas inferiores entran en senescencia y no ganan peso en vista de que se convierten en fuentes que atienden la demanda de fotoasimilados requeridos por las flores para su amarre y el posterior cuajado de frutos.

Aunque la porosidad y resistencia a la penetración variaron después de mecanizado el suelo, como se indica en la Tabla 2, esto no generó mayor incidencia en la TAN y la TCR de las plantas con relación a los tratamientos, lo cual puede estar relacionado con que la RP no superó los umbrales de compactación, donde pueden ocurrir problemas para el normal desarrollo de raíces (Botta et al., 2007).

\section{CONCLUSIONES}

Después de mecanizado el suelo, disminuyen los valores de Rp y Da, pero con el paso del tiempo y debido a diferentes factores, la tendencia de estas variables fue a incrementar, retornando a los valores iniciales reportados; Los rendimientos de papaya tienen una relación directa con la $\mathrm{Rp}$ y la $\mathrm{Da}$, siendo sensibles a impedancias mecánica de 1,1 MPa de Rp y 1,0 g/ $\mathrm{cm}^{3}$ de Da.

El Testigo no presentó compactación de acuerdo con los umbrales de porosidad y resistencia a la penetración. Después de aplicados los tratamientos disminuyó de forma notable la resistencia a la penetración y aumentó la porosidad.

El área foliar y la tasa de crecimiento del cultivo, fueron las variables más sensibles en respuesta de los cambios de porosidad y resistencia a la penetración del suelo.

El Testigo y el suelo mecanizado con azadón alcanzaron los mayores niveles de tasa de asimilación neta, y presentaron la mejor distribución de materia seca por unidad de área.

Las condiciones físicas de resistencia a la penetración y porosidad del suelo antes y después de la mecanización, estuvieron entre los rangos que no generan limitaciones para el desarrollo normal de las plantas.

\section{AGRADECIMIENTOS}

A la Vicerrectoría de Investigaciones y Posgrados de la Universidad de la Amazonia, por su apoyo logístico y financiero, al Semillero de Investigaciones en Agroecosistemas de la Amazonia (SIAM), por su esfuerzo, dedicación y trabajo en equipo. 


\section{REFERENCIAS BIBLIOGRAFÍCAS}

AGUILAR, L.; ESCALANTE, J.; FUCIKOVSKY, L.; TIJERINA, L.; ENGLEMAN, E. 2005. Área foliar, tasa de asimilación neta, rendimiento y densidad de población en girasol. Revista Terra Latinoamericana. 23(1):303 - 310.

ÁLVAREZ, C.; TABOADA, M.; BUSTINGORRI, C.; GUTIÉRREZ, B.; FLAVIO, H. 2006. Descompactación de suelos en siembra directa: efectos sobre las propiedades físicas y el cultivo de maíz. Revista Ciencia de Suelo. 24(1):1 - 10.

ÁLVAREZ, C.; TORRES, M.; CHAMORRO, E.; AMBROSIO, D.; TABOADA, M. 2009. Descompactación de suelos franco limosos en siembra directa: Efectos sobre las propiedades edáficas y los cultivos. Revista Ciencia del Suelo. 27(1):159 - 169.

ARROYO, L.; UGALDE, M.; ARAYA, E. 2003. Zonificación Agroecológica de 15 cultivos prioritarios de la Región Huetar Atlánica de Costa Rica, sus resultados y metodología a escala 1:50.000. Departamento de Suelos y Evaluación de Tierras. 55 - 58 p.

BARRAZA, F.; FISCHER, G.; CARDONA, C. 2004. Estudio del proceso de crecimiento del cultivo del tomate ( Lycopersicon esculentum Mill.) en el valle del Sinú Medio, Colombia. Revista Agronomía Colombiana. 22(1):81 - 90.

BARRERA, J.; SUÁREZ, D.; MELGAREJO, L. 2010. Análisis de crecimiento en plantas- Experimentos en Fisiología Vegetal. Primera Edición. Charlie's Impresores Ltda. Bogotá D.C. 247 p.

BELTRÁN, Y.; TORRIJO, R. 2013. Línea base de la industria láctea del Caquetá. En: Comité de Departamental de Ganaderos del Caquetá y Cámara de Comercio de Florencia. En: http//www.ccflorencia. org.co/descargas/informes $\% 20$ presidencia/2014/ L\%C3\%ADnea\%20Base \%20de\%20la\%20Industria\%20L\%C3\%A1ctea\%20del\%20Caquet\%C3\%A1. pdf; consulta: febrero, 2015.

BECERRA, C.; MADERO, E.; HERRERA, 0.; AMÉZQUITA, E. 2005. Caracterización espacial de la compactación en terrenos agrícolas de CIAT, Colombia. Revista del instituto de Investigación FIGMMG. 8(16): 33-37.

BORRERO, F.; FERNÁNDEZ, F.; LÓPEZ, A.; PARGA, V.; MURILLO, M.; CARVAJAL, A. 2000. Análisis de crecimiento en siete variedades de Papa (Solanum tuberosum L.). Revista Agronomía Mesoamericana. 11(1):145 - 149.
BOTTA, G.; POZZOLO, 0.; BOMBEN, M.; TOURN, M.; SOZA, E.; ROSATTO, H.; GILI, A.; RESSIA, J.; RIVERO, D.; VÁSQUEZ, J.; STADLER, S. 2007. Aplicación del tráfico controlado en la cosecha de maíz (Zea mays L.), Efecto sobre rendimientos del cultivo y las propiedades físicas del suelo. Revista Agro-Ciencia. 23(1):23 - 29.

BRAVO, C.; ANDREU, E. 1995. Propiedades físicas y producción de maíz (Zea mays L.) en un Alfisol del estado Guarico, Venezuela, bajo dos sistemas de labranza. Revista Venesuelos. 3(1):62 - 68.

CAMPOSTRINI, E.; YAMANISHI, 0. 2001. Influence of mechanical root restriction on gas-exchange of four papaya genotypes. Brazilian Journal of Plant Physiology. 13(1):129 - 138.

CAYÓN, G. 1992. Fotosíntesis y productividad de cultivos. Revista COMALFI. 19(1):23 - 31.

DELMONTE, R.; MAIA, J.; OLIVEIRA, O.; ROQUE, C. 2010. Uso de dois penetrômetros na avaliação da resistência mecânica de um latossolo vermelho distrófico. Revista Engenharia Agrícola e Ambiental. 30(1):307 - 314.

DÍAZ, Z. 2004. Efecto de la compactación subsuperficial de un hapludoltipico sobre la producción de girasol (Helianthus annus L.). Revista Ciencia del Suelo. 22(1):40 - 43.

DÍAZ, Y.; TORRES DE LA NOVAL, T.; RODRÍGUEZ, J.; RODRÍGUEZ, P. 2014. Respuesta de plantas de papaya silvestre (Carica cubensis Solms) al estrés hídrico y su recuperación: aspectos fisiológicos y del crecimiento. Cultivos Tropicales. 35(3): 55-61.

DI RIENZO, J.; CASANOVES, F; BALZARINI, M.; GONZÁLEZ, L.; TABLADO, M.; ROBLEDO, C. 2014. InfoStat versión 2014. Grupo InfoStat. En: FCA Universidad Nacional de Córdoba Argentina. En: http//www.infostat. com.ar; consulta: marzo, 2015.

ELISSONDO, E.; COSTA, J.; SUERO, E.; FABRIZZI, K.; GARCÍA, F. 2001. Evaluación de algunas propiedades físicas de suelos luego de la introducción de labranza verticales en suelo bajo siembra directa. Ciencia del suelo. 19(1): 11-19.

ESCAMILLA, J.; SAUCEDO, C.; MARTÍNEZ, T.; MARTÍNEZ, A.; SÁNCHEZ, P.; SOTO, R. 2003. Fertilización orgánica, mineral y foliar sobre el desarrollo y la producción de papaya cv. Maradol. Terra Latinoamericana. 21(2): 157-166. 
ESCOBAR, C.; ZULUAGA, J.; RIVERA, E. 1998. Aspectos de los suelos del departamento de Caquetá con relación al uso y manejo. Primera Edición. Fotomecánica Editorial. Caquetá, Colombia. 4 p.

FIRMINO, J.; PEREIRA, C.; DA SILVA, C. 2007. Índices fisiológicos e crescimento inicial de mamoeiro (Carica papaya L.) em casa de vegetação. Revista Ciência e Agrotecnologia. 31(1):1358 - 1363.

GARCÍA, J. 2006. Principios de agricultura orgánica: uso y manejo del suelo. Primera edición. Editorial JDC. Tunja, Colombia. 107-113 p.

GIL, A.; MIRANDA, D. 2007. Efecto de cinco sustratos sobre índices de crecimiento de plantas de papaya ( $\mathrm{Ca}$ rica papaya L.) bajo invernadero. Revista Colombiana de Ciencias Hortícolas, 1 (2):142 - 153.

GIL, C.; RODRÍGUEZ, G.; RAYMUNDO, J.; VELASCO, A.; CAMPOS, G. 2014. Índice de área foliar y factores de perturbación en una cuenca hidrográfica del rio Tehuantepec. Revista Mexicana de Agroecosistemas. 2(1):52 - 59 .

HERNÁNDEZ, R. 2009. Importancia socioeconómica del sector frutícola en Colombia. Trabajo de grado para optar al título de Administrador de Empresas Agropecuarias, Facultad de Ciencias Agricolas, Universidad de la Salle. Bogotá, Colombia. 82 p.

JARMA, A.; BUITRAGO, C.; GUTIÉRREZ, S. 1999. Respuesta del crecimiento de la habichuela (Phaseolus vulgaris L.) a tres niveles de radiación incidente. Revista COMALFI. 26(1):62 - 73.

JIMÉNEZ, J. 2002. Manual práctico para el cultivo de la papaya hawaiana. Primera edición. Earth. Costa rica. $108 \mathrm{p}$.

JORAJURIA, D.; DRAGHI, L. 2000. Sobre compactación del suelo agrícola Parte I: influencia diferencial del peso y del número de pasadas. Revista Brasileira de Engenharia Agricola e ambiental. 4(3):445 - 452.

MARTÍNEZ, D.; LANDINI, A.; SOZA, E.; HEREDIA, 0.; SAINATO, C. 2010. Efecto del pisoteo animal sobre las propiedades de un suelo. Parte I: densidad aparente, humedad, resistencia a la penetración, y modelos asociados. Agrociencia, Revista chilena ciencia agropecuaria. 27(1): 5-14.
MONTENEGRO, H.; MALANGÓN, D. 1990. Propiedades físicas de los suelos. Bogotá. IGAC. p 813.

PINZÓN, A.; AMÉZQUITA, E. 1991. Compactación de suelos por el pisoteo de animales en pastoreo en el piedemonte amazónico de Colombia. Revista Pasturas Tropicales. 13(1):21 - 26.

RICHART, A.; TAVARES, J.; RODRÍGUEZ, 0.; FUENTES, R.; FERREITA, R. 2005. Compactação do solo: causas e efeitos. Ciencias Agrarias, Londrina. 26 (3): 321-344.

RODRÍGUEZ, L.; CORCHUELO, G.; ÑUSTEZ, C. 2003. Influencia del espaciamiento entre plantas sobre la morfología y el crecimiento de la papa (Solanum tuberosum L.) bajo dos ambientes contrastantes. Revista Agronomía de Colombia. 21(1):210 - 219.

SANTOS, M.; SEGURA, M.; ÑUSTES, C. 2010. Análisis de crecimiento y relación fuente demanda de cuatro variedades de papa (Solanum tuberosum L.) en el municipio de Zipaquirá (Cundinamarca, Colombia). Revista Facultad Nacional de Agronomía. 63(1):253 - 266.

SOZA, E.; AGNES, D.; BOTTA, G.; TOURN, M.; HIDALGO, R. 2006. Descompactación del suelo mediante diferentes sistemas de labranza: su efecto sobre la emergencia y rendimiento en soja. Revista Agrotecnia. 16(1):22 - 27.

TABOADA, M. 1998. Compactación superficial causada por la siembra directa, y regeneración estructural en suelos franco limosos pampeanos. p 361-374. En: Memorias XVI Congreso Argentino de la Ciencia del Suelo. Rosario, Argentina.

TORRENTE, A.; VALENZUELA, B. 2013. Física de Suelos, pp. 65 - 95. En: Burbano, H. y Silva, Ciencia del Suelo: Principios Básicos/ Sociedad Colombiana de la Ciencia del Suelo. Segunda Edición. Offset Grafico Editores, Bogotá, Colombia. 304 p.

TRUJILLO, L.; CUELLAR, Y.; HUACA, D.; VELÁSQUEZ, J.; SUÁREZ, J. 2012. Caracterización de árboles dispersos en potreros y su efecto en la cobertura herbácea en pasturas del piedemonte amazónico Colombiano. Revista Momentos de Ciencia. 9(1):50 - 58.

YAMANISHI, 0.; CAMPOSTRINI, E.; MARTELLETO, M. 1998. Influence of root zone restriction on the growth of four papaya (Carica papaya L.) genotypes. Revista Acta Hort. 516(1):155 - 162. 